THE AstrophysicAL JoURNAL, 529:985-996, 2000 February 1

(C) 2000. The American Astronomical Society. All rights reserved. Printed in U.S.A.

\title{
A METHOD FOR DISTINGUISHING BETWEEN TRANSIENTLY ACCRETING NEUTRON STARS AND BLACK HOLES, IN QUIESCENCE
}

\author{
Robert E. Rutledge, ${ }^{1}$ Lars Bildsten, ${ }^{2}$ Edward F. Brown, ${ }^{3}$ George G. Pavlov, ${ }^{4}$ ANd Vyatcheslav E. Zavlin ${ }^{5}$ \\ Received 1999 May 20; accepted 1999 September 22
}

\begin{abstract}
Neutron stars and black holes often reside in binaries where the accretion rate onto the compact object varies by orders of magnitude. These "X-ray transients" are observed both in outburst (when the high accretion rate makes them X-ray bright) and quiescence (when the accretion rate is very low, or potentially zero). In a previous paper, we showed that the quiescent X-ray emission from three neutron star transients (Aq1 X-1, Cen X-4, and 4U 1608-522) were well represented by thermal emission from the neutron star's hydrogen atmosphere and that the emitting area was consistent with the whole surface. Previous blackbody spectral fits (which are not accurate representations of the thermal spectrum) severely underestimated the true emitting area. In this paper, we fit hydrogen atmosphere models to the X-ray data for four neutron stars (the three from the previous paper, plus $4 \mathrm{U} 2129+47$ ) and six black hole candidates (A0620-00, GS 2000+25, GS 1124-68, GS 2023+33, GRO J1655-40, and GRO $\mathrm{J} 0422+32$ ) with masses $\gtrsim 3 M_{\odot}$. While the neutron stars are similar in their intrinsic X-ray spectra (that is, similar effective temperatures and emission area radii $\sim 10 \mathrm{~km}$ ), the spectra of two black hole candidates are significantly different, and the spectra of the remaining four are consistent with a very large parameter space that includes the neutron stars. The spectral differences between the neutron stars and black hole candidates favors the interpretation that the quiescent neutron star emission is predominantly thermal emission from the neutron star surface. Higher quality data from Chandra, XMM, and $A S T R O-E$ will yield a much better contrast. There are many transients which do not have clear neutron star characteristics (such as type I X-ray bursts or coherent pulsations) and where the mass of the compact object is not constrained. In these cases, it is ambiguous as to whether the compact object is a neutron star or black hole. Our work suggests that an X-ray spectral comparison in quiescence provides an additional means for distinguishing between neutron stars and black holes. The faint X-ray sources in globular clusters - thought to be either cataclysmic variables or quiescent neutron stars - are a class of objects which can be investigated in this manner.

Subject headings: accretion, accretion disks - black hole physics - stars: atmospheres — stars: neutron
\end{abstract}

\section{INTRODUCTION}

Many black holes and neutron stars are in binaries where a steady state accretion disk (one that supplies matter to the compact object at the same rate as the mass is donated from the companion) is thermally unstable (Van Paradijs 1996; King, Kolb, \& Burderi 1996). This thermal instability results in a limit cycle - as in dwarf novae - with matter accumulating in the outer disk for $\sim$ months to decades until a thermal instability is reached (see Huang \& Wheeler 1989; Mineshige \& Wheeler 1989), triggering rapid accretion onto the compact object, and a substantial X-ray brightening (typically by $\times 10^{3}$ or more, up to $10^{38}$ ergs $\mathrm{s}^{-1}$ ). Both neutron star (NS) and black hole candidate (BHC) systems exhibit these X-ray outbursts, separated by periods ( $\sim$ months to decades) of relative quiescence (for recent reviews, see Tanaka \& Lewin 1995; Tanaka \& Shibazaki 1996; Chen, Shrader, \& Livio 1997; Campana et al. 1998a).

\footnotetext{
${ }^{1}$ Space Radiation Laboratory, California Institute of Technology, MS 220-47, Pasadena, CA 91125; rutledge@srl.caltech.edu.

${ }^{2}$ Institute for Theoretical Physics and Department of Physics, Kohn Hall, University of California, Santa Barbara, CA 93111; bildsten@itp.ucsb.edu.

${ }^{3}$ Department of Astronomy and Astrophysics, University of Chicago, 5640 South Ellis Avenue, Chicago, IL 60637; brown@flash.uchicago.edu.

${ }^{4}$ The Pennsylvania State University, 525 Davey Lab, University Park, PA 16802; pavlov@astro.psu.edu.

${ }^{5}$ Max-Planck-Institut für Extraterrestrische Physik, D-85740 Garching, Germany; zavlin@xray.mpe.mpg.de.
}

Deep pointed X-ray observations of these transients in quiescence have found that BHCs are, on average, less luminous in quiescence than their NS counterparts (Barret, McClintock, \& Grindlay 1996; Chen et al. 1997; Narayan, Garcia, \& McClintock 1997b; Asai et al. 1998). To explain the higher quiescent luminosities $\left(10^{32}-10^{33} \mathrm{ergs} \mathrm{s}^{-1}\right)$ of the transient NSs, Narayan et al. (1997b) suggested that matter continues to accrete during quiescence onto both objects and that neutron stars reradiate the infalling matter's kinetic energy, while black holes swallow most of the accreted mass energy. In this picture, the required quiescent accretion rate, $\dot{M}_{q}$, onto the compact object in BHC systems must be substantially greater than in NS systems. Current spectral modeling (for an advection dominated flow) of the few X-ray detected BHCs use $\dot{M}_{q}$ of $\sim \frac{1}{3}$ of the total mass transfer rate in the binary (Narayan, Barrett, \& McClintock 1997a), whereas the more efficient energy release onto a neutron star requires that $\dot{M}_{q}$ be smaller by $2-3$ orders of magnitude. The cause of this difference in $\dot{M}_{q}$ between the BHC and NS systems is not easily explained (Menou et al. 1999).

An alternative picture for the NS emission was put forward by Brown, Bildsten, \& Rutledge (1998), who showed that NSs radiate a minimum luminosity, even if accretion completely ceases during quiescence. This minimum luminosity comes from energy deposited in the inner crust (at a depth of $\sim 300 \mathrm{~m}$ ) during the large accretion events. The freshly accreted material compresses the inner crust and triggers nuclear reactions that deposit 
$\approx \mathrm{MeV}$ per accreted baryon there (Haensel \& Zdunik 1990). This heats the NS core on a $10^{4}-10^{5} \mathrm{yr}$ timescale, until it reaches a steady state temperature $\approx 4 \times 10^{7}\left(\langle\dot{M}\rangle / 10^{-11}\right.$ $\left.M_{\odot} \mathrm{yr}^{-1}\right)^{0.4} \mathrm{~K}$ (Bildsten \& Brown 1997), where $\langle\dot{M}\rangle$ is the time-averaged accretion rate in the binary. A core this hot makes the NS incandescent, at a luminosity $L_{q}=1$ $\mathrm{MeV}\langle\dot{M}\rangle / m_{p} \approx 6 \times 10^{32}\left(\langle\dot{M}\rangle / 10^{-11} M_{\odot} \mathrm{yr}^{-1}\right) \mathrm{ergs} \mathrm{s}^{-1}$, even after accretion halts (Brown et al. 1998). The NS is then a thermal emitter in quiescence, much like a young NS.

For both hypotheses, the energy source for the quiescent luminosities of the BHCs and NSs have different physical causes, making it meaningful to search for distinguishing spectral signatures.

\subsection{X-Ray Spectra of Quiescent Neutron Stars}

The first NS transient detected in quiescence was Cen X-4 (Van Paradijs et al. 1987). More recently, quiescent X-ray spectral measurements have been made of Aql X-1 (Verbunt et al. 1994) and 4U 2129+47 (Garcia \& Callanan 1999 ) with the ROSAT/PSPC; of EXO $0748-676$ with the Einstein IPC (Garcia \& Callanan 1999); and of Cen X-4 and 4U 1608-522 with $A S C A$ (Asai et al. 1996b). The $\mathrm{X}$-ray spectrum of Aq1 X-1 (0.4-2.4 keV) was consistent with a blackbody (BB) spectrum, a bremsstrahlung spectrum, or a pure power-law spectrum (Verbunt et al. 1994). For $4 \mathrm{U} 1608-522$, the spectrum $(0.5-10.0 \mathrm{keV})$ was consistent with a $\mathrm{BB}\left(k T_{\mathrm{BB}} \approx 0.2-0.3 \mathrm{keV}\right)$, a thermal RaymondSmith model $\left(k T=0.32_{-0.5}^{+0.18} \mathrm{keV}\right)$, or a very steep power law (photon index $6_{-2}^{+1}$ ). Similar observations of Cen X-4 with $A S C A$ found its $\mathrm{X}$-ray spectrum consistent with these same models, but with an additional power-law component (photon index $\approx 2.0$ ) above $5.0 \mathrm{keV}$ (recent observations with BeppoSAX of Aql X-1 in quiescence also revealed a power-law tail; Campana et al. 1998b). The origin of the observed power-law spectral components in Cen X-4 and Aql X-1 is not clear. While it has been suggested they may be due to magnetospheric accretion (Campana et al. 1998a), spectral models of metallic NS atmospheres (Rajagopal \& Romani 1996; Zavlin, Pavlov, \& Shibanov 1996) also predict hard tails. These warrant further observational investigation. The observation of EXO $0748-676$ shows it to be more luminous (by $\times 10-50$ ) than the other four NSs.

In four of these five sources (the exception being EXO 0748-676), BB fits implied an emission area of radius $\approx 1 \mathrm{~km}$, much smaller than a NS. This had little physical meaning however, as the emitted spectrum from a quiescent NS atmosphere with light elements at the photosphere is far from a blackbody. For a weakly magnetic $\left(B \leq 10^{10} \mathrm{G}\right)$ pure hydrogen or helium ${ }^{6}$ atmosphere at effective temperatures $k T_{\text {eff }} \lesssim 0.5 \mathrm{keV}$ the opacity is dominated by freefree transitions (Rajagopal \& Romani 1996; Zavlin et al. 1996). Because of the opacity's strong frequency dependence $\left(\propto v^{-3}\right)$, higher energy photons escape from deeper in the photosphere, where $T>T_{\text {eff }}$ (Pavlov \& Shibanov 1978; Romani 1987; Zampieri et al. 1995). Spectral fits of the Wien tail (which is the only part of the spectrum sampled with current instruments) with a BB curve then overesti-

\footnotetext{
${ }^{6}$ The strong surface gravity will quickly (within $\sim 10 \mathrm{~s}$ ) stratify the atmosphere (Alcock \& Illarionov 1980; Romani 1987); for accretion rates $\lesssim 2 \times 10^{-13} M_{\odot} \mathrm{yr}^{-1}$ (corresponding to an accretion luminosity $\lesssim 2$ $\times 10^{33} \mathrm{ergs} \mathrm{s}^{-1}$ ), metals will settle out of the photosphere faster than the accretion flow can supply them (Bildsten, Salpeter, \& Wasserman 1992). As a result, the photosphere should be nearly pure hydrogen.
}

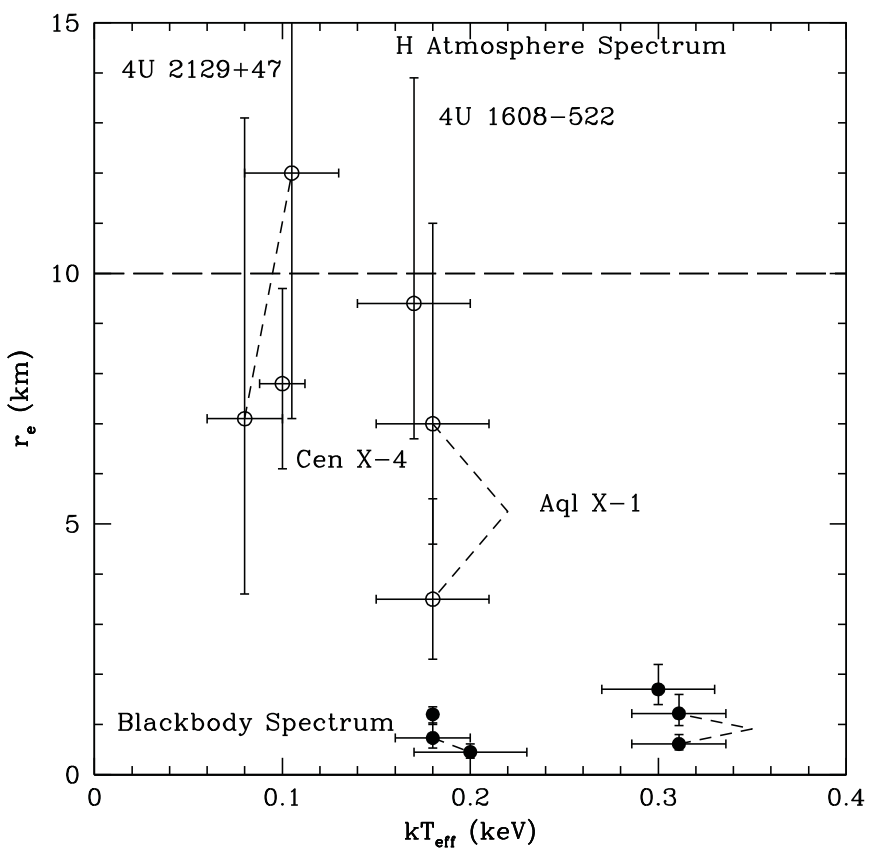

FIG. 1.-Comparison between the spectral parameters $r_{e}$ and $k T_{\text {eff }}$, derived from spectral fits of the quiescent X-ray emission from Aql X-1, Cen X-4, 4U 1608-522, and 4U 2129+47. The open circles are for the $\mathrm{H}$ atmosphere spectrum, and the filled circles are from a blackbody spectrum. The two points connected for Aql X-1 correspond to the upper and lower distance limits for that source. The two connected points for 4U 2129+47 are for the two different distance $/ N_{\mathrm{H}}$ estimates (see text). As expected from comparisons between the theoretical spectra, the $\mathrm{H}$ atmosphere fits produce higher values of $r_{e}$.

mate $T_{\text {eff }}$ and underestimate the emitting area, by as much as orders of magnitude (Rajagopal \& Romani 1996; Zavlin et al. 1996). ${ }^{7}$

Rutledge et al. (1999) showed that fitting the spectra of quiescent NS transients with these models yielded emitting areas consistent with a $10 \mathrm{~km}$ radius NS. In Figure 1, we compare the measured $\mathrm{H}$ atmosphere and blackbody spectral parameters for the quiescent NSs. The data for $4 \mathrm{U} 2129+47$ are analyzed here, while the other sources were analyzed previously (Rutledge et al. 1999). The emission area radii are larger from the $\mathrm{H}$ atmosphere spectra by a factor of a few to 10 and are consistent with the canonical radius of a NS. There is thus both observational evidence and theoretical motivation that thermal emission from a pure hydrogen photosphere contributes to - and perhaps dominates - the NS luminosity at photon energies at $0.1-1$ $\mathrm{keV}$. This makes possible the use of quiescent NS X-ray spectra as an astrophysical tool.

\subsection{Quiescent $X$-Ray Spectroscopy of NSs and BHCs}

Distinguishing between a stellar-mass black hole and a neutron star in an X-ray binary is a nontrivial observational problem. Although there are X-ray phenomena unique to NSs (for example, type I X-ray bursts and coherent pulsations), as yet, no X-ray phenomenon predicted to

\footnotetext{
${ }^{7}$ Application of $\mathrm{H}$ atmosphere models to the isolated neutron stars in SNR PKS 1209-52 and Puppis A produced a source distance consistent with that measured through other means (assuming a $10 \mathrm{~km}$ NS radius), a lower surface temperature, and an X-ray measured column density that was consistent with that measured from the extended SNR (while the column density measured with an assumed BB spectrum was not consistent with other measurements; Zavlin et al. 1998, 1999).
} 
occur exclusively in BHs has been observed. In the absence of any distinctive NS properties, some X-ray transients are classified as BHCs if they display X-ray spectral and variability properties similar to those of other BHCs, such as $\sim 30 \%$ rms frequency-band-limited variability accompanied by a hard spectrum, or 3-12 Hz quasi-periodic oscillations while the source has high X-ray intensity, although there are NS systems which display these properties as well (see Van der Klis 1995 for a review). While a statistical distinction between the quiescent luminosities of BHCs and NSs has been demonstrated (see references in $\S 1$ ), there is overlap in the observed luminosities; thus, while the different average quiescent luminosities support the hypothesis that the (preclassified) objects belong to distinct classes, quiescent luminosity cannot be used to distinguish between a NS and a $\mathrm{BHC}$ on a case-by-case basis. A promising phenomenological distinction between NSs and BHCs is that, at X-ray luminosities greater than $10^{37} \mathrm{ergs} \mathrm{s}^{-1}$, the 20-200 keV luminosity of BHCs is systematically higher than that of NSs. However, the physical origin of this difference is not known, and the weakness of a phenomenological distinction is its vulnerability to a single counterexample (Barret et al. 1996).

By far the most solid technique is measuring or constraining the mass of the compact object via radial velocity measurements of the optical companion. If the resulting optical mass function indicates that the compact object mass exceeds $3 M_{\odot}$, then it is likely to be a black hole, as the mass limit of a NS has been calculated to be below $3.2 M_{\odot}$ (Rhoades \& Ruffini 1974; Chitre \& Hartle 1976). The large amount of progress in this method has given us several very secure black holes (McClintock 1998).

A new spectroscopic distinction between transient NSs and $\mathrm{BHs}$ - based on the presence of a neutron star's photosphere - would be a valuable classification tool. We present the first such comparisons here, where we report a spectral analysis that uses an accurate emergent spectrum from a NS to fit the quiescent X-ray spectra of six transient BHCs with measured mass functions (GRO J0422+32, A0620-00, GS 1124-68, GRO J1655-40, GS 2000+25, and GS $2023+33$ ) and four transient neutron stars.

We begin in $\S 2$ by describing the BHCs and NSs we have chosen for this comparison, as well as the hydrogen atmosphere models. Three of the NS are from our previous study (Rutledge et al. 1999), Aq1 X-1, Cen X-4, 4U 1608-522; and we discuss a fourth here, $4 \mathrm{U} 2129+47$. We show in $\S 3$ that although the neutron stars occupy a narrow range of effective temperatures and emitting area radii, no such relation is found among the BHCs. This implies that the $\mathrm{H}$ atmosphere spectrum may be used as a tool to distinguish between NSs and BHCs in quiescence (in the absence of other information). Section 4 discusses the state of our observational understanding of the energy source for the quiescent emission from the NSs. We conclude in $\S 5$ by summarizing and briefly discussing the application of this work to X-ray sources in globular clusters.

\section{OBJECT SELECTION AND DATA ANALYSIS}

Our purpose in fitting the $\mathrm{H}$ atmosphere modelappropriate only for NSs - to the BHC data is to directly compare the measured spectral parameters of the BHCs to those of NSs. We selected BHCs for analysis from among those in Table 1 of Menou et al. (1999), which contains a list of eight compact binary systems with implied masses $\gtrsim 3 M_{\odot}$. Three of these systems (A0620-00, GS 2023+33, and GRO J1655-40) were detected in X-rays in quiescence (at luminosities $\lesssim 10^{33} \mathrm{ergs} \mathrm{s}^{-1}$ ), the data for which we analyze; the remaining five have upper limits in their luminosity. We analyze the three with luminosity upper limits below $10^{33} \mathrm{ergs} \mathrm{s}^{-1}$ (GRO J0422+32, GS $2000+25$, and GS 1124-68) to investigate the constraints on emission area radius as a function of surface temperature. The remaining two sources with high-luminosity upper limits $\geq 10^{33} \mathrm{ergs} \mathrm{s}^{-1}$ (N Oph 1977 and 4U 1543-47) are consistent with or greater than the luminosities from NSs (Rutledge et al. 1999), and therefore they cannot be excluded as NSs based on a spectral comparison; we do not investigate them here.

Brief descriptions of the analyzed observations are in Table 1. Data were obtained from the public archive at HEASARC/GSFC. ${ }^{8}$ All observations analyzed are listed in Table 1; all were performed with ROSAT/PSPC, except for those of GRO J1655-40, which were performed with $A S C A$, and one observation of GRO J0422 + 32, which was performed with ROSAT/HRI.

For our spectral fits and derived parameters, we adopted column densities using the conversion of $N_{\mathrm{H}, 22} \equiv N_{\mathrm{H}} / 10^{22}$ $\mathrm{cm}^{-2}=0.179 A_{V} \quad$ (Predehl \& Schmitt 1995) and $A_{V}=$ $3.1 E(B-V)$ (Schild 1977), except where noted. We extracted the data for each source as described in the Appendix, and fitted each extracted spectrum with a spectral model of galactic absorption and a tabulated $\mathrm{H}$ atmosphere model (Zavlin et al. 1996), using XSPEC (Arnaud 1996).

The $\mathrm{H}$ atmosphere model is determined by two spectral parameters: an effective temperature $\left(k T_{\text {eff }}\right)$ and the ratio of an emission area radius $\left(r_{e}\right.$; the true radius, which would be measured as the circumference of the NS, divided by $2 \pi$ ) to source distance. In our results, we quote $r_{e}$ by assuming a source distance (which can be uncertain by a factor of 2 , and therefore represents a considerable systematic uncertainty in both the NSs and BHCs). The $\mathrm{H}$ atmosphere spectrum we used assumes the surface gravity of a $1.4 M_{\odot}, 10 \mathrm{~km}$ object. It is possible to adopt the surface gravity as an additional parameter to the $\mathrm{H}$ atmosphere model; however, the available data are of insufficient quality to constrain simultaneously all three parameters.

We discuss in the Appendix the column densities adopted for each individual source. In quiescence, the $\mathrm{S} / \mathrm{N}$ of the data is typically not sufficient to measure the X-ray equivalent column density for the assumed spectrum. We thus adopt an historically measured value of $N_{\mathrm{H}}$, taken either from: (1) X-ray absorption observed while the object is X-ray bright; (2) optical reddening, which has been measured proportional to the equivalent hydrogen column density; or (3) the neutral hydrogen column density from radio observations (Dickey \& Lockman 1990) taken from the W3NH tool at HEASARC, ${ }^{9}$ which measures the integrated column density not just to the distance of the X-ray object, but through the galaxy. All of these methods, when applied to estimating the $N_{\mathrm{H}}$ during X-ray quiescence, have systematic errors sufficient to produce more than $100 \%$ uncertainties in the implied emission area and surface temperature (see discussion in Rutledge et al. 1999). In addition, there are observations suggesting a column density that varies over timescales of months to years, between out-

\footnotetext{
${ }^{8} \mathrm{http}: / /$ heasarc.gsfc.nasa.gov/.

${ }^{9}$ http://heasarc.gsfc.nasa.gov/Tools/Tools.html.
} 
TABLE 1

OBSERVATIONS

\begin{tabular}{|c|c|c|c|c|}
\hline Number & Satellite/Instrument & $\begin{array}{l}\text { Observation } \\
\text { Start Time } \\
\text { (UT) }\end{array}$ & $\begin{array}{l}\text { Live Time } \\
\quad(\mathrm{ks})\end{array}$ & $\begin{array}{c}\text { Average Count } \\
\text { Rate }^{\mathrm{a}} \\
\text { (counts s }^{-1} \text { ) }\end{array}$ \\
\hline \multicolumn{5}{|c|}{ GRO J0422+32 } \\
\hline $1 \ldots \ldots$. & $R O S A T / \mathrm{PSPC}$ & 1993 Aug 17 04:37 & 28.0 & $(8.0 \pm 0.2) \times 10^{-2}$ \\
\hline $2 \ldots \ldots$ & $R O S A T / \mathrm{HRI}$ & 1995 Jun 6 12:29 & 18.8 & $(3.3 \pm 3.3) \times 10^{-4}$ \\
\hline \multicolumn{5}{|c|}{ A0620-00 } \\
\hline $3 \ldots \ldots \ldots$ & $R O S A T / \mathrm{PSPC}$ & 1992 Mar 10 17:05 & 29.8 & $(1.5 \pm 0.3) \times 10^{-3}$ \\
\hline \multicolumn{5}{|c|}{ GS $1124-68$} \\
\hline $4 \ldots \ldots \ldots$ & $R O S A T / \mathrm{PSPC}$ & 1992 Mar 1 00:11 & 16.0 & $(4.0 \pm 3.5) \times 10^{-4}$ \\
\hline \multicolumn{5}{|c|}{ GRO J1655-40 } \\
\hline $5 \ldots \ldots \ldots$ & ASCA & 1996 Mar 23 00:54 & 87.0 & $(2.1 \pm 0.4) \times 10^{-3}(\mathrm{SIS} 1)$ \\
\hline \multicolumn{5}{|c|}{ GS $2000+25$} \\
\hline $6 \ldots \ldots$ & ROSAT/PSPC & 1992 May 1 13:23 & 12.4 & $(0.8 \pm 6.0) \times 10^{-4 b}$ \\
\hline $7 \ldots \ldots$ & ROSAT/PSPC & 1993 Apr 9 06:41 & 6.6 & $\ldots$ \\
\hline $8 \ldots \ldots \ldots$ & ROSAT/PSPC & 1993 Oct 13 13:11 & 6.8 & $\ldots$ \\
\hline \multicolumn{5}{|c|}{ GS $2023+33$} \\
\hline $9 \ldots \ldots$ & $R O S A T$ & 1992 Nov 5 13:04 & 16.5 & $(2.1 \pm 0.1) \times 10^{-2}$ \\
\hline \multicolumn{5}{|c|}{$4 U 2129+47$} \\
\hline $10 \ldots \ldots$ & $R O S A T / \mathrm{HRI}$ & 1991 Dec 9 12:47 & 7.0 & $(4.8 \pm 1.0) \times 10^{-3}$ \\
\hline $11 \ldots \ldots$ & $R O S A T / \mathrm{PSPC}$ & 1994 Mar 6 00:10 & 31.0 & $(6.7 \pm 0.5) \times 10^{-3}$ \\
\hline
\end{tabular}

${ }^{a}$ Count-rates are background subtracted. For ROSAT/PSPC and HRI, count rates are for the energy range $0.4-2.4 \mathrm{keV}$; for $A S C A$ the relevant instrument is listed.

${ }^{\mathrm{b}}$ Average for observations 6,7, and 8 .

bursts of transients. An example is a change of $\Delta N_{\mathrm{H}}=0.5$ $\times 10^{22} \mathrm{~cm}^{-2}$ in $4 \mathrm{U} 1608-522$ (Penninx et al. 1989) over several months. This uncertainty in $N_{\mathrm{H}}$ can only be overcome by high $\mathrm{S} / \mathrm{N}$ data in $\mathrm{X}$-ray quiescence, which can permit a direct measurement of $N_{\mathrm{H}}$ during the observation. Interpretation of the results of the present analysis must bear this systematic uncertainty in mind.

\section{COMPARISON BETWEEN BLACK HOLE CANDIDATES AND NEUTRON STARS IN QUIESCENCE}

In Figure 2, we compare the measured spectral parameters $k T_{\text {eff }}$ and $r_{e}$ of quiescent NSs and BHCs. Error bars are $90 \%$ confidence, as are upper limits. For the four NSs in this analysis, the $k T_{\text {eff }}$ and $r_{e}$ were constrained, with best-fit $k T_{\text {eff }}$ in the range $0.08-0.20 \mathrm{keV}$, and $r_{e}$ in the range $8-12 \mathrm{~km}$. The NSs exhibit a significant range in temperature, possibly due to different core temperatures (related to $\langle\dot{M}\rangle$ ). The data are of sufficient quality to constrain $r_{e}$ to within a factor of 2 for the NSs (not accounting for the uncertainties in source distance), and the resulting $r_{e}$ are consistent with objects of $10 \mathrm{~km}$ radius. The two connected points for Aql X-1 are for two different distances $(2.0$ and $4.0 \mathrm{kpc})$. The two connected points for $4 \mathrm{U} 2129+47$ are discussed in the analysis $\S \mathrm{A} 7$. We note that recent observations (Callanan, Filippenko, \& Garcia 1999) have resolved the optical counterpart of Aq1 X-1 into two objects, only one (at most) will be associated with the X-ray system; most likely, this observation implies a distance to Aq1 X-1 greater than previous estimates, although a new distance estimate has not yet been produced.
The spectra of five of the six BHCs were of insufficient statistics to simultaneously constrain both $r_{e}$ and $k T_{\text {eff }}$ of the $\mathrm{H}$ atmosphere model. The exception (GS $2023+33$ ) was significantly harder than the NS spectra, which constrained the $k T_{\text {eff }}$ to be above those observed from the NSs. The BHCs GRO J1655-40, GS $2000+25$, GRO J0422+32, and GS $1124-68$ have spectral parameters that are, within errors, consistent with those of the NSs. A0620-00 and GRO J0422+32, if we presumed these to be NSs of the same surface area and gravity, must be cooler $(\lesssim 0.05 \mathrm{keV})$ than the average observed transient NSs. The spectrum of A0620-00 is discrepant with those of the NSs, implying substantially smaller emission area $\left(r_{e} \sim 0.2-2 \mathrm{~km}\right)$ for the range of $k T_{\text {eff }}$ observed from the NSs.

The addition of a power-law spectral component-such as the hard power-law tail as observed from Cen X-4 in $A S C A$ data, and detected in Aql X-1 in BeppoSAX dataintroduces enough uncertainty in the $\mathrm{H}$ atmosphere spectral parameters of BHCs that all sources would be consistent with those we observe from the NSs. In noting this systematic uncertainty, we point out that additional spectral components are not demanded by the data we have used, although the data is largely of low bandwidth (0.4-2.4 $\mathrm{keV}$ ), and that wider bandwidth data (such as from $A S C A$, Chandra, or $X M M$ ) may alter this. In practice, we have neglected this systematic uncertainty to investigate the question: if these BHC sources were discovered in quiescence, and their $\mathrm{H}$ atmosphere spectral parameters were compared with those from known NSs, would we conclude they are similar or dissimilar to the NSs? For GS 2023+33 


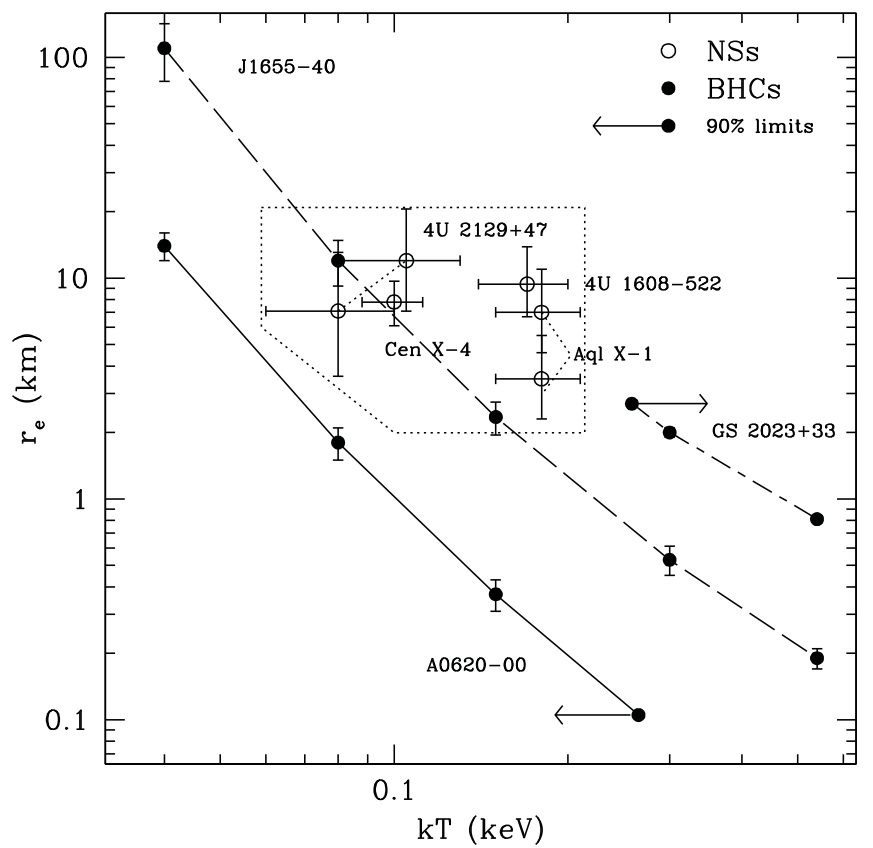

FIG. 2.-Best-fit emission area radii for NSs and BHCs (A0620-00, GS $2023+33$, GRO J1655-40) using a hydrogen atmosphere spectrum, with the surface gravity of a $1.4 M_{\odot}, 10 \mathrm{~km}$ object. The dotted-line box encloses the (phenomenological) parameter space occupied by the NSs. The $90 \%$ upper limits for GS 2000+25, GS 1124-68, and GRO $\mathrm{J} 0422+32$ are not included (see Table 3) but are lines which lie between GRO J1655-40 and A0620-00 across $k T_{\text {eff }}=0.04-0.054 \mathrm{keV}$, passing through the NS box. The best-fit parameters for the NSs cluster in the $k T_{\text {eff }} \sim 0.08-0.2 \mathrm{keV}$ and $r_{e} \sim 8-12 \mathrm{~km}$. The best-fit parameters for BHCs GRO J1655-40, GS 1124-68, GS $2000+25$, and GRO J0422 + 32 are consistent with having similar spectra, while GS $2023+33$ and A0620-00 are inconsistent.

and A0620-00, we find that they are dissimilar; while for GRO J1655-40, GS1124-683, GS2000+25, and GRO $\mathrm{J} 0422+32$, we find they are consistent (albeit with a wide range of values as well). Higher signal-to-noise data, taken with an instrument of wider bandwidth, would permit us greater certainty regarding the possible contributions of spectral parameters for which we have not here accounted.

Moreover, no BHC has well-constrained spectral parameters which would place it exclusively within the parameter space occupied by the NSs, as depicted on Figure 2. This is a phenomenologically defined region, which would have to be expanded with the discovery of NSs in quiescence outside of this box. Thus, we find no evidence that any of the BHCs have been misclassified and should be reclassified as NSs.

\section{THE ENERGY SOURCE FOR THE NEUTRON STAR QUIESCENT LUMINOSITY}

There are presently no observational results which exclude that part of the quiescent luminosity of these NSs is due to accretion. Brown et al. (1998) noted a few observational tests which can be applied to determine whether accretion is active. First, as accretion will increase source luminosity (at this low luminosity level), the spectra should be drawn when sources are at their lowest observed luminosity in the X-ray passband. Second, since the $\mathrm{H}$ atmosphere thermal flux is expected to be variable only on timescales of longer than $\sim$ months to $10^{4} \mathrm{yr}$, variability on timescales less than this ( $\lesssim$ days) likely indicates active acc- retion. Second, active accretion onto the NS surface will produce metal absorption lines in the spectrum $(\mathrm{O}$ and $\mathrm{Fe}$, below $1 \mathrm{keV}$ ), which can be observed with Chandra and $X M M$, but which cannot be observed with $A S C A$ or ROSAT. The presence of photospheric absorption metal lines in the spectrum - aside from being observationally important-will indicate active accretion onto the NS surface. These indicators should be used to insure that the observed NS emission is not due to active accretion (in addition to being observationally consistent with the theoretical $\mathrm{H}$ atmosphere spectrum). Since present instrumentation is not capable of detecting the lines, we can only apply the variability and observed luminosity criteria.

If accretion is occurring during quiescence, this will increase the $k T_{\text {eff }}$ of an emergent $\mathrm{H}$ atmosphere spectrum and produce metal absorption lines; accretion will not affect $r_{e}$ unless emission originates from a different surface than the NS (such as in an accretion disk).

Some observational evidence suggests that accretion is indeed occurring onto the NS surface during quiescence; long-term (months to years) variability in the observed flux has been reported (for $4 \mathrm{U} 2129+47$, see $\S \mathrm{A} 7.1$ and Garcia \& Callanan 1999; for Cen X-4, see Van Paradijs et al. 1987). While this variability can be explained by a variable absorption column depth, active accretion during quiescence is also a possibility. However, recent observations of Aql X-1 at the end of an outburst showed an abrupt fading into quiescence (Campana et al. 1998b) associated with a sudden spectral hardening (Zhang, Yu, \& Zhang 1998a). This was followed by a period of $\sim 15$ days, over which the source was observed (three times) with a constant flux level (Campana et al. 1998b). This behavior was interpreted as the onset of the "propeller effect" (Illarionov \& Sunyaev 1975; Stella, White, \& Rosner 1986) in this object, which would inhibit - perhaps completely - accretion from the disk onto the NS. The energy source for the long-term nearly constant flux is then a puzzle. Unlike the accretionpowered models, the work of Brown et al. (1998) makes a specific prediction for this constant flux level, relating it to the long term time-averaged accretion rate.

So as to minimize any contributions from accretion, we only analyze observations made during periods of the lowest observed flux. We calculate the bolometric luminosity from the $\mathrm{H}$ atmosphere fits. The fits are given in terms of an (unredshifted) effective temperature $\left(k T_{\text {eff }}\right)$ and emitting area radius $\left(r_{e}\right)$. The observed bolometric flux is $F_{\text {bol }}=(1+z)^{-2}\left(r_{e} / d_{0}\right)^{2} \sigma T_{\text {eff }}^{4}$, where $d_{0}$ is the source distance, and the observed bolometric luminosity is $L_{\mathrm{bol}}=$ $4 \pi d_{0}^{2} F_{\text {bol }}$. For a NS of $1.4 M_{\odot}$ and $10 \mathrm{~km}$ radius, the surface redshift is $1+z=\left(1-2 G M / R c^{2}\right)^{-1 / 2}=1.31$. In Table 4 we give the unabsorbed, observed X-ray and bolometric luminosities assuming a $\mathrm{H}$ atmosphere spectrum and the surface redshift of a $1.4 M_{\odot} 10 \mathrm{~km}$ radius NS $(1+z=1.31)$, measured as described in the analysis of the present work and previously (Rutledge et al. 1999). The luminosity quoted for $4 \mathrm{U} 1608-522$ in the previous work was not calculated for the distance stated there; the correct value is in this table. While there is considerable systematic uncertainty in these values, due to uncertain distances and $N_{\mathrm{H}}$, the luminosities cover an order of magnitude, at about $10^{32-33}$ ergs s$^{-1}$.

Using these new bolometric quiescent luminosities for Aql X-1, Cen X-4, and 4U 1608-522, we have remade a plot (Fig. 3) of $L_{q} / L_{o}$ as a function of $t_{r} / t_{o}$ (Brown et al. 


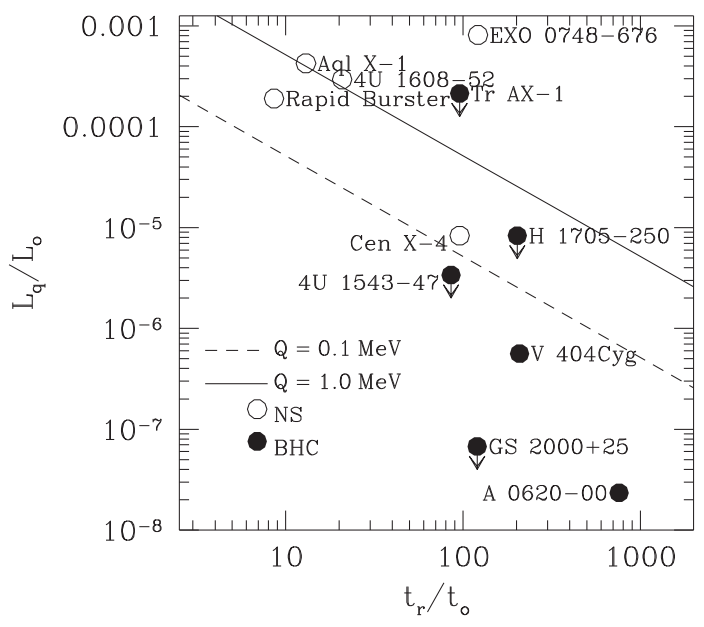

FIG. 3.-Ratio of quiescent luminosity $L_{q}$ to outburst luminosity $L_{o}$ as a function of the ratio of recurrence interval $t_{r}$ to outburst duration $t_{o}$. The lines are for different amounts of heat, $0.1 \mathrm{MeV}$ (dashed line) and $1.0 \mathrm{MeV}$ (solid line), per accreted nucleon deposited at depths where the thermal time is longer than the outburst recurrence time. Also plotted are the observed ratios for several NSs (filled circles) and BHs (open circles). For most of the BHs, only an upper limit (arrow) to $L_{q}$ is known. Data are from Chen et al. (1997), with the exception of $L_{q}$ for the Rapid Burster (Asai et al. 1996b). For Aql X-1 and the Rapid Burster, $L_{o}$ and $t_{o}$ are accurately known ( $R X T E /$ All-Sky Monitor public data); for the remaining sources $L_{o}$ and $t_{o}$ are estimated from the peak luminosities and the rise and decay timescales. For Aq1 X-1, Cen X-4, and 4U 1608-522, we have recalculated the bolometric luminosities using the $\mathrm{H}$ atmosphere models (Rutledge et al. 1999).

1998). Here $L_{q}$ and $L_{o}$ are the observed quiescent and average outburst luminosities, and $t_{r}$ and $t_{o}$ are the recurrence interval and outburst duration. We show this relation for the NSs (open circles) Aq1 X-1, Cen X-4, 4U 1608-522, and EXO 0748-676 and the BHCs (filled circles) H 1705-250, 4U 1543-47, Tra X-1, V 404 Cyg (GS 2023+33), GS 2000+25, and A 0620-00. We denote with an arrow those BHCs for which only an upper limit on $L_{q}$ is known. Because the recurrence time for the NS 4 U $2129+47$ is unknown, we do not show it here. The expected incandescent luminosity is plotted for two different amounts of heat per accreted nucleon stored in the core during an outburst: $1 \mathrm{MeV}$ (solid line) and $0.1 \mathrm{MeV}$ (dotted line). The use of bolometric quiescent luminosities moved Aql X-1, Cen X-4, and 4U 1608-522 to higher $L_{q} / L_{o}$ (upward on this diagram). With the exception of these three objects and the Rapid Burster ( $L_{q}$ is from Asai et al. 1996a), the data from this plot are taken from Chen et al. (1997). For Aq1 X-1 and the Rapid Burster, $L_{o}$ and $t_{o}$ are accurately known (RXTE/All-Sky Monitor public data); for the remaining sources $L_{o}$ and $t_{o}$ are estimated from the peak luminosities and the rise and decay timescales.

Four of the five NSs are within the band where the quiescent luminosity is that expected when the emitted heat is between 0.1-1.0 MeV per accreted baryon. The fifth NS (EXO 0748-676), has a higher quiescent luminosity (by a factor of 10), which we interpret as being due to continued accretion, an interpretation which is reinforced by the observation of spectral variability during the quiescent observations with ASCA (Corbet et al. 1994; Thomas et al. 1997), on timescales of $\sim 1000 \mathrm{~s}$ and longer. (Garcia \& Callanan 1999 measured $L_{\mathrm{X}}=1 \times 10^{34}$ ergs $\mathrm{s}^{-1}$ from Einstein/IPC observations of this source.) The BHCs on this figure are more spread out across the parameter space, qualitatively indicating a statistical difference-although not one which is particular for each object-between the two classes of objects. This suggests the NS quiescent luminosity is more strongly related to the accreted energy than the $\mathrm{BH}$ quiescent luminosities.

\section{CONCLUSIONS}

We have fitted the quiescent X-ray spectra of four transient NSs and six transient BHCs (with measured mass functions) with a pure $\mathrm{H}$ atmosphere spectrum. We compared the emitting area radius and effective temperature of the 10 sources and found that the NSs are clustered in $\left(r_{e}, k T_{\text {eff }}\right)$ parameter space. Two of the BHCs (A0620-00, GS 2023+33) are inconsistent with the NS spectra; the upper limits of three more (GS 1124-68, GS 2000+25, GRO J0422+32), and the $\left(r_{e}, k T_{\text {eff }}\right)$ locus of the sixth (GRO J1655-40) overlap the NS parameter space. The upper limits of the parameter space of GS $2000+25$ and GRO J0422+32 are marginally consistent with the observed $\left(r_{e}, k T_{\text {eff }}\right)$ of the NSs, indicating that these BHCs, if interpreted as NSs, would have to be cooler than the average NS observed in quiescence.

We found that the X-ray spectra excludes A0620-00 and GS $2023+33$ from being NSs of the type we used for comparison. These differences between the quiescent X-ray spectra of BHCs and NSs cannot be directly attributed to an observational selection effect. The NSs were identified by the type I X-ray bursts, the BHCs were first identified by their X-ray variability behavior and later by the high $(\gtrsim 3$ $M_{\odot}$ ) implied mass of the compact object. The X-ray spectra of GS $1124-68$, GS $2000+25$, GRO J1655-40, and GRO $\mathrm{J} 0422+32$ are consistent with those of the NSs, but their parameter space is large, and better data are needed to determine whether they are spectrally similar or not.

Our method can be applied to objects which have low $\mathrm{X}$-ray luminosities $\left(\lesssim 10^{34}\right.$ ergs $\left.\mathrm{s}^{-1}\right)$, to identify them as neutron stars, which have an atmosphere from which the theoretical emission originates, or as some object which is not a NS, as we have done here. In addition to the transient field objects, this method can be readily applied to the lowluminosity X-ray sources observed in globular clusters (Hertz \& Grindlay 1983), which are thought to be cataclysmic variables (Cool et al. 1995; Grindlay et al. 1995) but may also be transient neutron stars in quiescence (Verbunt, Elson, \& Van Paradijs 1984). X-ray spectroscopic determination can identify these objects as NSs radiating thermal emission from the atmosphere or imply a different origin for the emission. As discussed above and elsewhere (Brown et al. 1998), the quiescent luminosities of these sources are set by the time average accretion rate. Thus, the low-luminosity $\left(10^{31}\right.$ ergs s$\left.^{-1}\right) \mathrm{X}$-ray sources in globular clusters, if they were transient neutron stars in quiescence, would have $\langle\dot{M}\rangle \approx 2 \times 10^{-13} M_{\odot} \mathrm{yr}^{-1}$; comparing this to Aql X-1, with $\langle\dot{M}\rangle \approx 1 \times 10^{-10} M_{\odot} \mathrm{yr}^{-1}$ (estimated from the $R X T E / A S M$ light curve history), and a mean outburst interval of $\approx 200$ days, the low-luminosity X-ray sources would have Aql-like outbursts with recurrence times of $\sim 250 \mathrm{yr}$, assuming the quiescent luminosity is in steady state with accretion. The estimated number of such sources down to this luminosity level is highly uncertain, about 1-10 per globular cluster (Verbunt et al. 1995). For $\sim 200$ GCs in the galaxy, one expects $\sim 1-10$ such transients per year. Even assuming only $\frac{1}{4}$ of all GCs are within distance to detect an Aq1 X-1-like transient with the RXTE/ASM 
TABLE 2

OBJECT INFORMATION

\begin{tabular}{|c|c|c|c|}
\hline Object & $\begin{array}{c}d_{0} \\
(\mathrm{kpc})\end{array}$ & $\begin{array}{l}\text { Mass } \\
\left(M_{\odot}\right)\end{array}$ & $\begin{array}{c}N_{\mathrm{H}} \\
\left(10^{22} \mathrm{~cm}^{-2}\right)\end{array}$ \\
\hline \multicolumn{4}{|c|}{ Black Hole Candidates with Mass Estimates } \\
\hline GRO J0422+32 ..... & $2.6^{\mathrm{a}}$ & $>9^{\mathrm{b}}$ & $0.22^{\mathrm{c}}$ \\
\hline $\mathrm{A} 0620-00 \ldots \ldots \ldots . .$. & $0.9^{\mathrm{d}}$ & $>7.3^{\mathrm{e}}$ & $0.22^{\mathrm{d}, \mathrm{f}}$ \\
\hline GS $1124-68 \ldots \ldots \ldots$ & $3.3^{\mathrm{g}}$ & $5.0-7.5^{\mathrm{h}}$ & $0.16^{\mathrm{i}}$ \\
\hline 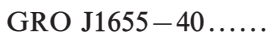 & $3.2^{\mathrm{j}}$ & $7.02 \pm 0.22^{\mathrm{k}}$ & $0.70^{1}$ \\
\hline GS $2000+25 \ldots \ldots \ldots$ & $2.0^{\mathrm{m}}$ & $7.0-7 . \overline{7} \pm 0.5^{\mathrm{n}}$ & $0.66-1.14^{\circ}$ \\
\hline GS $2023+33 \ldots \ldots \ldots$ & $3.5^{\mathrm{p}}$ & $8-12^{p}$ & $0.57^{q}$ \\
\hline \multicolumn{4}{|c|}{ Neutron Star } \\
\hline $4 \mathrm{U} 2129+47^{\mathrm{r}}$. & $1.5^{\mathrm{s}}$ & (NS) & $0.28^{\mathrm{s}}$ \\
\hline $4 \mathrm{U} 2129+47^{\mathrm{t}} \ldots$ & $6.0^{\mathrm{t}}$ & (NS) & $0.17^{\mathrm{t}}$ \\
\hline
\end{tabular}

Notes.-Conversion factors: $N_{\mathrm{H}, 22}=0.179 A_{V} ; A_{V}=3.1 E(B-V)$. See text.

${ }^{\text {a }}$ Esin et al. 1998.

b Beekman et al. 1997.

${ }^{c}$ Shrader et al. 1992.

d Oke \& Greenstein 1977.

e McClintock \& Remillard 1986.

${ }^{\mathrm{f}}$ Wu et al. 1976.

g Shahbaz et al. 1997.

${ }^{\text {h }}$ Orosz et al. 1996.

${ }^{\text {i }}$ Ebisawa et al. 1994.

${ }^{j}$ Hjellming \& Rupen 1995.

k Orosz \& Bailyn 1997.

${ }^{1}$ W3NH; Dickey \& Lockman 1990; Ueda et al. 1998; Horne et al. 1996.

${ }^{\mathrm{m}}$ Chevalier \& Ilovaisky 1990.

${ }^{\mathrm{n}}$ Casares et al. 1995.

o W3NH; Dickey \& Lockman 1990; Chevalier \& Ilovaisky 1990; Tsunemi et al. 1989.

p Wagner et al. 1992.

q Wagner et al. 1991.

r Thorstensen et al. 1979, Chevalier et al. 1989.

s Thorstensen et al. 1979.

${ }^{t}$ Cowley \& Schmidtke 1990.

(for a peak $R X T E /$ ASM detection count rate of 10 counts $\mathrm{s}^{-1}$; Aq1 X-1 has an ASM peak count rate of $\sim 30$ counts $\mathrm{s}^{-1}$ ), this produces an expected transient discovery rate of $0.3-3$ per year, which is consistent with or greater than the observed discovery rate with $R X T E / A S M$ (of no sources over a 4 yr period). Stronger constraints could be made with more sensitive all-sky monitoring observations over longer time-baselines, or with a more tightly constrained luminosity function of the low-luminosity sources in globular clusters.

Higher quality X-ray data from the coming X-ray spectroscopy missions (Chandra/ACIS, XMM, and ASTRO-E) will permit this analysis to be performed with greater accuracy, in particular by permitting the simultaneous measurement of the X-ray column density - a dominant systematic uncertainty. These will also provide the means to account for possible contributions due to a hard-power law component in the BHCs. For example, a 15 ks observation with Chandra of a source with photon power-law slope of 2, and luminosity of $2 \times 10^{31} \mathrm{ergs} \mathrm{s}^{-1}, N_{\mathrm{H}, 22}=0.2$ at $1 \mathrm{kpc}$ would produce a spectrum which can be excluded as a $\mathrm{H}$ atmosphere (or blackbody) with an (unconstrained) column depth, with probability $=6 \times 10^{-6}$. Finally, the high spectral resolution and count rates of these instruments will permit a search for short timescale variability and photospheric metal absorption lines, which would indicate ongoing accretion during X-ray quiescence (Brown et al. 1998).

This research was supported by NASA via grant NAGW4517 and through a Hellman Family Faculty Fund Award (UC-Berkeley) to L. B., who is also a Cottrell Scholar of the Research Corporation. E. F. B. is supported by NASA GSRP Graduate Fellowship under grant NGT 5-50052. G. G. P. acknowledges support from NASA grants NAG 5-6907 and NAG 5-7017. This research was supported in part by the National Science Foundation under grant PHY 94-07194. We acknowledge use of data obtained through the High Energy Astrophysics Science Archive Research Center Online Service, provided by the NASA/ Goddard Space Flight Center. We gratefully acknowledge useful conversations with D. Fox and A. Prestwich regarding $R O S A T / \mathrm{HRI}$ response issues. We gratefully acknowledge helpful comments from J. McClintock and from the referee, J. Grindlay.

\section{APPENDIX A}

\section{SOURCES}

The assumed distances (used to calculate luminosity) and adopted $N_{\mathbf{H}}$ for the spectral fits performed here are listed in Table 2; values for the NSs we analyzed previously (Aq1 X-1, Cen X-4, 4U 1608-522) are in the previous reference (Rutledge et al. 1999). The results of the spectral fits are presented in Table 3, which contains: (1) the data set number (cf. Table 1); (2) the $N_{\mathrm{H}}$; (3) the best-fit spectral parameters for the $\mathrm{H}$ atmosphere model, including the un-redshifted effective NS surface temperature $\left(k T_{\text {eff }}\right)$ and apparent emission area radius $r_{e}(\mathrm{~km})$, as well as the reduced $\chi_{v}^{2}$ for that model. The $\mathrm{H}$ atmosphere modelappropriate only for NSs - is applied here to the BHCs, to produce spectral parameters which can then be directly compared with those observed from NSs.

Here, we describe our assumptions about individual objects and provide specifics of the analyses of each observation.

\section{A1. GRO J0422+32}

The color excess, measured from an IUE spectrum of this object, was found to be $E(B-V)=0.40 \pm 0.07\left(N_{\mathbf{H}, 22} \sim 0.23\right.$ \pm 0.04; Shrader, Wagner, \& Starrfield 1992).

First, we analyze observation 1, a ROSAT/PSPC observation which had not previously been analyzed (this observation is included for completeness, as this source was fainter during observation 2). We extracted the data from a 53" circle about the source, and background from a 530" annulus about the source, excluding another object in the FOV, and three low surface brightness areas clustered in the SE side of the annulus (possibly background fluctuations). Due to the long exposure, this 
TABLE 3

Derived Spectral Parameters

\begin{tabular}{|c|c|c|c|c|}
\hline $\begin{array}{l}\text { Data Set } \\
\text { Number }\end{array}$ & $\begin{array}{c}N_{\mathrm{H}} \\
\left(10^{22} \mathrm{~cm}^{-2}\right)\end{array}$ & $\begin{array}{l}k T_{\text {eff }} \\
(\mathrm{keV})\end{array}$ & $\begin{array}{c}r_{e} \\
{\left[\mathrm{~km}\left(d / d_{0}\right)\right]}\end{array}$ & $\begin{array}{c}\chi^{2} \\
(\mathrm{dof})\end{array}$ \\
\hline \multicolumn{5}{|c|}{ GRO J0422 + 32} \\
\hline $1 \ldots \ldots \ldots \ldots \ldots$ & $\begin{array}{r}<0.12 \\
(0.22)\end{array}$ & $\begin{array}{l}0.14 \pm 0.01 \\
0.14 \pm 0.01\end{array}$ & $\begin{array}{r}3_{-0.7}^{+2.4} \\
11_{-2.5}^{+4.7}\end{array}$ & $\begin{array}{l}1.64 / 1 \\
6.3 / 2\end{array}$ \\
\hline $2 \ldots \ldots \ldots \ldots \ldots$ & $\begin{array}{l}(0.22) \\
(0.22) \\
(0.22) \\
(0.22) \\
(0.22)\end{array}$ & $\begin{array}{l}(0.04) \\
(0.08) \\
(0.15) \\
(0.30) \\
(0.54)\end{array}$ & $\begin{array}{l}<27 \\
<3.6 \\
<0.8 \\
<0.2 \\
<0.08\end{array}$ & $\begin{array}{l}\mathrm{n} / \mathrm{a} \\
\mathrm{n} / \mathrm{a} \\
\mathrm{n} / \mathrm{a} \\
\mathrm{n} / \mathrm{a} \\
\mathrm{n} / \mathrm{a}\end{array}$ \\
\hline \multicolumn{5}{|c|}{ A0620-00 } \\
\hline 3 ….......... & $\begin{array}{l}(0.22) \\
(0.22) \\
(0.22) \\
(0.22)\end{array}$ & $\begin{array}{l}(0.04) \\
(0.08) \\
(0.15) \\
(0.265)\end{array}$ & $\begin{array}{l}14 \pm 2 \\
1.8 \pm 0.3 \\
0.37 \pm 0.06 \\
0.105\end{array}$ & $\begin{array}{l}1.2 / 2 \\
0.3 / 2 \\
1.9 / 2 \\
4.0 / 2\end{array}$ \\
\hline \multicolumn{5}{|c|}{ GS $1124-68$} \\
\hline $4 \ldots \ldots \ldots \ldots \ldots$ & $\begin{array}{l}(0.16) \\
(0.16) \\
(0.16) \\
(0.16) \\
(0.16) \\
\end{array}$ & $\begin{array}{l}(0.04) \\
(0.08) \\
(0.15) \\
(0.30) \\
(0.54) \\
\end{array}$ & $\begin{array}{l}<82 \\
<8.6 \\
<1.2 \\
<0.26 \\
<0.13\end{array}$ & $\begin{array}{l}\mathrm{n} / \mathrm{a} \\
\mathrm{n} / \mathrm{a} \\
\mathrm{n} / \mathrm{a} \\
\mathrm{n} / \mathrm{a} \\
\mathrm{n} / \mathrm{a}\end{array}$ \\
\hline \multicolumn{5}{|c|}{ GRO J1655-40 } \\
\hline 5 .............. & $\begin{array}{l}(0.74) \\
(0.74) \\
(0.74) \\
(0.74) \\
(0.74)\end{array}$ & $\begin{array}{l}(0.04) \\
(0.08) \\
(0.15) \\
(0.30) \\
(0.54)\end{array}$ & $\begin{aligned} 110 & \pm 32 \\
12.0 & \pm 2.8 \\
2.35 & \pm 0.4 \\
0.53 & \pm 0.08 \\
0.19 & \pm 0.02\end{aligned}$ & $\begin{array}{r}105 / 70 \\
100 / 70 \\
95 / 70 \\
80 / 70 \\
61 / 70\end{array}$ \\
\hline \multicolumn{5}{|c|}{ GS $2000+25$} \\
\hline 6,7, and $8 \ldots \ldots$ & $\begin{array}{l}(0.66) \\
(0.66) \\
(0.66) \\
(1.1) \\
(0.66) \\
(0.66)\end{array}$ & $\begin{array}{l}(0.04) \\
(0.08) \\
(0.15) \\
(0.15) \\
(0.30) \\
(0.54)\end{array}$ & $\begin{array}{l}<28 \\
<3.8 \\
<1.0 \\
<1.9 \\
<0.29 \\
<0.13\end{array}$ & $\begin{array}{l}\mathrm{n} / \mathrm{a} \\
\mathrm{n} / \mathrm{a} \\
\mathrm{n} / \mathrm{a} \\
\mathrm{n} / \mathrm{a} \\
\mathrm{n} / \mathrm{a} \\
\mathrm{n} / \mathrm{a}\end{array}$ \\
\hline \multicolumn{5}{|c|}{ GS $2023+33$} \\
\hline 9 .............. & $\begin{array}{l}(0.54) \\
(0.54) \\
(0.54)\end{array}$ & $\begin{array}{l}(0.258) \\
(0.30) \\
(0.54)\end{array}$ & $\begin{array}{l}2.7 \pm 0.1 \\
2.0 \pm 0.1 \\
0.81 \pm 0.03\end{array}$ & $\begin{array}{l}4.0 / 2 \\
1.8 / 2 \\
0.72 / 2\end{array}$ \\
\hline \multicolumn{5}{|c|}{$4 \mathrm{U} 2129+47\left(1.5 \mathrm{kpc} ; N_{\mathrm{H}, 22}=0.28\right)$} \\
\hline $11 \ldots \ldots \ldots \ldots$ & $(0.28)$ & $0.08_{-0.015}^{+0.02}$ & $7.1_{-3.5}^{+6.0}$ & $0.13 / 1$ \\
\hline \multicolumn{5}{|c|}{$4 \mathrm{U} 2129+47\left(6.0 \mathrm{kpc} ; N_{\mathrm{H}, 22}=0.17\right)$} \\
\hline $11 \ldots \ldots \ldots \ldots \ldots$ & $(0.17)$ & $0.105_{-0.020}^{+0.025}$ & $12_{-4.9}^{+8.6}$ & $0.71 / 1$ \\
\hline
\end{tabular}

NOTES.-Errors and upper limits are $90 \%$ confidence; values listed in parenthesis were fixed during the fits. For description of the different $\left(d_{0}, N_{\mathrm{H}}\right)$ assumptions for $4 \mathrm{U} 2129+47$, see $\S \mathrm{A} 7$.

spectrum constrained all three parameters $\left(N_{\mathrm{H}}, k T_{\text {eff }}\right.$, and $\left.r_{e}\right)$. The resulting best-fit was $r_{e}=3.0_{-0.7}^{+2.4} \mathrm{~km}, k T_{\text {eff }}=0.22_{-0.05}^{+0.03}$ $\mathrm{keV}$, and $N_{\mathrm{H}, 22}<0.12(90 \%)$, for an implied luminosity of $7.8 \times 10^{32}(d / 2.6 \mathrm{kpc})^{2} \mathrm{ergs} \mathrm{s}^{-1}{ }^{e}(0.5-2.0 \mathrm{keV})$. In a fit holding the column density fixed at $N_{\mathrm{H}, 22}=0.22$, the best-fit model was not acceptable at the $p=1.8 \%$ level. The parameter space for a $\Delta \chi^{2}=2.72$ would constrain the $r_{e}$ to be similar to those found from the NSs $\left(11_{-2.5}^{+4.7} \mathrm{~km}\right)$.

We analyzed the HRI observation 2 . The observation was previously analyzed, producing a luminosity upper limit of $\log (L)<31.6\left(d=2.6 \mathrm{kpc}, \alpha=2.1\right.$, and $N_{\mathrm{H}, 22}=0.2$; Garcia et al. 1997). We extracted the spectrum from an $8^{\prime \prime}$ radius about the source position - finding seven photons, consistent with the expected and measured background count rate. We extracted background counts from an annulus of 50" and 10" outer and inner radii, respectively. We used the 1990 December 1 HRI spectral response from GSFC/HEASARC calibration database. We find a slightly lower, but consistent $3 \sigma$ upper limit to the unabsorbed source luminosity for the same assumed spectrum $(\log (L)<31.4)$ as found previously. We assumed a series of 
temperatures and found upper limits to $r_{e}$ for the assumed spectrum. For a comparable temperature as derived from the PSPC analysis (above), the implied $r_{e}<0.8 \mathrm{~km}$ - substantially below that found from the PSPC analysis, which indicates intensity variability at this low luminosity. As observation 2 has lower luminosity than observation 1 , we use observation 2 in our interpretations (as thermal emission from the NS surface should not vary by $\gtrsim$ few percent over the timescales here, and it is only the lowest observed luminosity from each object which may be due in its greatest part to the thermal surface emission).

\section{A2. $\mathrm{A} 0620-00$}

The optical color excess of the companion star has been measured as $E(B-V)=0.39 \pm 0.02$ (Wu et al. 1976; Oke \& Greenstein 1977), corresponding to $N_{\mathrm{H}, 22}=0.22$-somewhat below the $N_{\mathrm{H}, 22}=0.41$ (Dickey \& Lockman 1990) measured in this direction (which is the integrated value along this line of sight through the entire galaxy), consistent with a nearby object. We adopt the $N_{\mathrm{H}, 22}=0.22$ value for our spectral fits.

We analyzed data taken by ROSAT/PSPC (observation 3); these data were previously analyzed (McClintock, Horne, \& Remillard 1995). An X-ray source was found in the extracted data at the source position. Counts from this source were extracted from a circle of radius 53". Background was taken from an annulus about the source, $4^{\prime}$ outer radius, and $60^{\prime \prime}$ inner radius; excluded from this annulus were circular areas (each only half-within the annulus, straddling the outer radius) about two unrelated sources, each with radius 53". The source region contained a total of 116 counts. The background region contained 938 counts. We used data in the energy range $0.4-2.1 \mathrm{keV}$, rebinned into three energy bins $0.4-0.8,0.8-1.2$, and $1.2-2.1 \mathrm{keV}$. Using the same spectral model and assumptions as the previous work (McClintock et al. 1995), we reproduce the best-fit blackbody temperature and source luminosity.

The data are of insufficient $\mathrm{S} / \mathrm{N}$ to constrain the temperature and area independently. We held the temperature fixed at values $k T_{\text {eff }}=[0.04,0.54] \mathrm{keV}$, and extracted the best-fit emission area radii $\left(r_{e}\right)$, which were found to range from $14 \pm 2 \mathrm{~km}$ (at $\left.k T_{\text {eff }}=0.04 \mathrm{keV}\right)$ to $0.07 \mathrm{~km}\left(\right.$ at $\left.k T_{\text {eff }}=0.15 \mathrm{keV}\right)$. The model $\chi_{v}^{2}$ reaches a probability of $2 \%$ that the observed spectrum is produced by the model in a single random trial $\left(\chi_{v}^{2}=4.0\right.$, for 2 dof $)$ at $k T_{\text {eff }}=0.265 \mathrm{keV}$, which sets our upper limit on the effective temperature for this source.

\section{A3. GS $1124-68$}

The X-ray measured equivalent column density was found to have evolved, decreasing by $N_{\mathrm{H}, 22} \sim 0.2$ during the outburst, and settling to a value of $N_{\mathrm{H}, 22}=0.16$ (Ebisawa et al. 1994), consistent with the optical reddening $E(B-V)=0.20 \pm 0.05$ (Gonzalez-Riestra et al. 1991) measured from a UV absorption line, and is below the measured hydrogen column density in the direction of this source $N_{\mathbf{H}, 22}=0.25$ (Dickey \& Lockman 1990), consistent with an object a short distance away relative to the size of the galaxy. We adopt $N_{\mathrm{H}, 22}=0.16$ for our spectral fits.

The PSPC observation (number 4) has been previously analyzed (Greiner et al. 1994; Narayan et al. 1997b). For the source, we extracted counts from a $75^{\prime \prime}$ radius circle about the source position, as the source position is about $15^{\prime}$ off-axis. For background, we used three circular areas on the detector, one centered at the source position, the other two centered at distances from the FOV center equal to that of the source position. From these three circular areas, we excluded the $75^{\prime \prime}$ region about the source position, and one other apparent (that is, faint) source. There were 66 counts in the source region, and 2104 counts in the background spectrum. We generated an ancillary response file for this off-axis spectrum using the FTOOL pcarf V2.1.0. In the spectral analyses, we find a $3 \sigma$ upper limit to the (unabsorbed) source flux of less than $3.5 \times 10^{-14} \mathrm{ergs}$ $\mathrm{cm}^{-2} \mathrm{~s}^{-1}(0.3-2.4 \mathrm{keV})$, assuming $N_{\mathrm{H}, 22}=0.22$, and a power-law photon spectral slope of $\alpha=2.5$, consistent with the previously found value.

There is no detectable quiescent source at the position of the optical source, consistent with previous results (Greiner et al. 1994; Narayan et al. 1997b). Across the investigated range of NS surface temperatures, the upper limits of the implied radii range from 82 to $0.13 \mathrm{~km}$.

\section{A4. GRO J1655-40}

This $A S C A$ observation has been analyzed previously (Ueda et al. 1998), in which the flux was measured to be $2 \times 10^{-13}$ ergs $\mathrm{cm}^{-2} \mathrm{~s}^{-1}(2-10 \mathrm{keV})$. The interstellar column density was measured at $N_{\mathrm{H}, 22}=0.74 \pm 0.03$ in the X-ray high (bright) state, and less than 0.08 and less than $0.14(90 \%)$ in the low (faint) state. The optical reddening is $E(B-V)=1.3 \pm 0.1$ (cf. Orosz \& Bailyn 1997; Horne et al. 1996) is consistent with a value of $N_{\mathrm{H}, 22}=0.74$, which we adopt and hold fixed.

Using data taken with the GIS in PH mode, we extracted background counts from two circles, each $5^{\prime}$ in radius and centered $5^{\prime}$ from the image center-the same distance as the position of the object. The source X-ray spectra were extracted from a $5^{\prime}$ circle about the optical position. We excluded energy bins below $1.0 \mathrm{keV}$ and above $5.0 \mathrm{keV}$ from the fit.

For the SIS, data were taken from 2.5 radius circles about the source. Background was taken from a square approximately $6^{\prime}$ on a side, excluding the $2^{\prime} .5$ source region. We used energy range of $1.0-10.0 \mathrm{keV}$ for the GIS data and $0.5-10.0 \mathrm{keV}$ for the SIS data.

Based on these data, no single best fit is found for $0.04 \mathrm{keV}<k T_{\text {eff }}<0.54 \mathrm{keV}$. As the assumed temperature (held fixed) is increased from $k T_{\text {eff }}=0.04$ to $0.54 \mathrm{keV}, r_{e}$ decreases from $110 \pm 32 \mathrm{~km}$ to $0.19 \pm 0.02 \mathrm{~km}$.

\section{A5. GS $2000+25$}

The X-ray equivalent $N_{\mathrm{H}}$ for this object has been measured as $N_{\mathrm{H}, 22}=1.14$ (Tsunemi et al. 1989), and optical reddening measured as $E(B-V)=1.5$ (Chevalier \& Ilovaisky 1990) (corresponding to $N_{\mathrm{H}, 22}=0.825$ ), which are slightly discrepant from one another, though within the systematic uncertainties between these techniques. The measured hydrogen column density in the direction of this object is $N_{\mathrm{H}, 22}=0.66$ (Dickey \& Lockman 1990), although within a degree, values range between 
$N_{\mathrm{H}, 22}=[0.51,0.81]$, and at the most proximate point, $N_{\mathrm{H}, 22}=0.67$. The higher measured optical reddening of the stellar companion and absorption of X-rays from the compact object, relative to the direct $N_{\mathrm{H}}$ measurement, may indicate significant absorption in the local environment of the binary, on the order of $N_{\mathrm{H}, 22}=0.5$. We use in turn $N_{\mathbf{H}, 22}=0.66$ and $N_{\mathrm{H}, 22}=1.1$.

Two of the presently analyzed observations (6 and 7) have been analyzed previously (Verbunt et al. 1994). In the ROSAT image for any of the three observations $(6,7$, and 8$)$ there is no detected object at the source position. We combine the data, which are of three widely varying epochs, to produce average upper limits to spectral parameters.

In each of the three observations, we extract source counts from a circular region centered on the object of $60^{\prime \prime}$ radius. Background counts were taken from annuli about the source, with an inner radius of 63".75 and an outer radius of $375^{\prime \prime}$. We also excluded two circular regions (60" radius) which overlapped the annuli, which appear to contain sources. There were a total of 78 counts in the source region, and 2505 counts in the background region. The resultant spectrum produces flux upper limits consistent with (but below, by about 25\%) the higher upper limits found previously (Verbunt et al. 1994).

We do not detect a source above the background count rate; we produce $90 \%$ upper limits to $r_{e}$, which were found to range from 0.13 to $28 \mathrm{~km}$, depending on the assumed surface temperature, in the range of $0.04-0.54 \mathrm{keV}$.

\section{A6. GS $2023+33$}

The optical reddening has been measured at $A_{V} \sim 3.0$ (Charles et al. 1989), and a comparable $E(B-V)=1.03$ (Wagner et al. 1991), which imply $N_{\mathrm{H}, 22}=0.54-0.59$. This is below the measured $N_{\mathrm{H}}=0.81$ in this direction (Dickey \& Lockman 1990) consistent with an object nearby relative to the size of the galaxy. We adopt a value of $N_{\mathrm{H}, 22}=0.54$.

This object was observed twice in quiescence: in 1994 with $A S C A$ and in 1992 with ROSAT/PSPC (see Verbunt et al. 1994). It was also observed and analyzed in the ROSAT All-Sky Survey (Verbunt et al. 1994), on November 5, with an average count rate of $0.028 \pm 0.009$ counts s$^{-1}$, in which it was found with a luminosity of about $1.5 \times 10^{33}(d / 3.5 \mathrm{kpc})^{2} \mathrm{ergs} \mathrm{s}^{-1}(0.4-2.4$ $\mathrm{keV})$.

From the ROSAT image, data were selected centered on the X-ray source, in a circle $60^{\prime \prime}$ in radius. Background was taken from an annulus with an inner radius of 63.8 and an outer radius of $375^{\prime \prime}$. The source region had 425 counts, and the background region had a total of 1365 counts.

At effective temperatures below, $k T_{\text {eff }}=0.258$, the model becomes untenable $\left(\chi_{v}^{2}=4.0,2\right.$ dof, corresponding to $98 \%$ probability), which sets the lower limit of the acceptable effective temperature parameter space for this data. The best-fit area was not well constrained from below (as the temperature increases to values above those for which this model is valid), but the $90 \%$ upper limit on the radius is $1.9 \mathrm{~km}(\mathrm{~d} / 3.5 \mathrm{kpc})$. We find the $(0.4-2.4 \mathrm{keV})$ luminosity of the best-fit model to be $6 \times 10^{32}$ $\left(d / 3.5 \mathrm{kpc}^{2} \mathrm{ergs} \mathrm{s}^{-1}\right.$.

These results are not consistent with those of an earlier analysis of these same data (Wagner et al. 1994), whose analysis produces a source flux that is 10 times greater than ours. We attribute this difference to Wagner et al.'s higher column density $\left(N_{\mathrm{H}, 22}=1.7\right.$ vs. our 1.5$)$, which we find is not motivated by the data. We note that intensity variability was found on $\sim$ days timescales, which a priori cannot be attributed to the NS surface flux from the hot (thermal) core. These results are also consistent with results from $A S C A$ analysis of quiescent data taken at a different time (Narayan et al. 1997a).

\section{A7. $4 \mathrm{U} 2129+47$}

We inadvertently did not include this NS transient in our previous study, so we analyze it here. This spectrum has been analyzed previously with a blackbody model (Garcia \& Callanan 1999), and the derived parameters were consistent with those we find here, under similar distance/column density assumptions.

The distance to this object is controversial with values between 1 and $6 \mathrm{kpc}$ (see Cowley \& Schmidtke 1990 for arguments on both sides). Associated with this distance uncertainty is an optical reddening uncertainty; if the optical counterpart is more distant, it must be more luminous, of earlier type, and bluer, in which case the reddening is $E(B-V)=0.3\left(N_{\mathrm{H}, 22}=0.17\right.$; Cowley \& Schmidtke 1990); if less distant, the companion is redder, and $E(B-V)=0.5\left(N_{\mathrm{H}, 22}=0.28\right.$; Thorstensen et al. 1979; Chevalier et al. 1989). The total $N_{\mathrm{H}}$ measured in the direction of this object is $N_{\mathrm{H}, 22}=0.38$ (Dickey \& Lockman 1990), while the best-fit column density obtained by Garcia \& Callanan (with these same data; Garcia \& Callanan 1999) was $N_{\mathrm{H}, 22}=21.5$ (no quoted error).

In interpreting the data, we alternately adopt a distance of $1.5 \mathrm{kpc}$ and $E(B-V)=0.5\left(N_{\mathrm{H}, 22}=0.28\right)$, and $6.0 \mathrm{kpc}$ and $E(B-V)=0.3\left(N_{\mathrm{H}, 22}=0.17\right)$.

For the 1994 March PSPC observation, the source counts were extracted from a circular region 45" in radius, with a total 279 counts. Background was extracted from an annulus about the source, $278^{\prime \prime}$ and $45^{\prime \prime}$ in outer and inner radius, respectively, for a total of 1959 counts. We fitted to data in the $0.4-2.4 \mathrm{keV}$ energy range.

For an assumed $N_{\mathrm{H}, 22}=0.28(d=1.5 \mathrm{kpc})$, the best-fit parameters were $k T_{\text {eff }}=0.08_{-0.015}^{+0.02} \mathrm{keV}$, and $r_{e}=7.1_{-3.5}^{+6.0} \mathrm{~km}$. For $N_{\mathrm{H}, 22}=0.17(d=6.0 \mathrm{keV})$, the best-fit parameters are $k T_{\text {eff }}=0.105_{-0.020}^{+0.025}$, and $r_{e}=12.0_{-4.9}^{+8.6}$. The unabsorbed luminosities for the assumed $\left(N_{\mathrm{H}}, d\right)$ combinations are $6.5 \times 10^{31}$ and $5.6 \times 10^{32} \mathrm{ergs} \mathrm{s}^{-1}(0.4-2.4 \mathrm{keV})$, respectively (see Table 4).

\section{A7.1. An Observed Change in the Flux from $4 U 2129+47$}

To compare the observed flux of $4 \mathrm{U} 2129+47$ analyzed here, with one measured previously with the ROSAT/HRI during a 7 ks of observations (1992 November-December; Garcia et al. 1997), we imposed a $N_{\mathrm{H}, 22}=0.5$ and a photon power-law slope of 2.0, and measured the (absorbed) flux from the 1994 March observation, which was $(6.7 \pm 0.5) \times 10^{-14} \mathrm{ergs} \mathrm{cm}^{-2}$ $\mathrm{s}^{-1}(0.3-2.4 \mathrm{keV}$ ) - a factor of $3.4 \pm 0.6$ below that measured during the earlier observation by Garcia (we independently analyzed the earlier HRI observation, and confirm Garcia's flux measurement). We cannot simultaneously extract $r_{e}$ and $k T_{\text {eff }}$ from the HRI observation, as only one spectral bin is available, and so we cannot compare the photon energy spectra. However, we jointly fitted both observed spectra with the same $\mathrm{H}$ atmosphere and $N_{\mathrm{H}}$ and found that there is a $2.7 \%$ chance 
TABLE 4

Bolometric Luminosity of Transient NeUtron Stars in Quiescence

\begin{tabular}{lcc}
\hline \hline \multicolumn{1}{c}{ Source } & $\begin{array}{c}L_{\mathrm{X}}(0.5-10.0 \mathrm{keV}) \\
\left(10^{32} \mathrm{ergs} \mathrm{s}^{-1}\right)\end{array}$ & $\begin{array}{c}L_{\mathrm{bol}} \\
\left(10^{32} \mathrm{ergs} \mathrm{s}^{-1}\right)\end{array}$ \\
\hline Aq1 X-1 & $(20.4 \pm 9.2)\left(d_{0} / 4 \mathrm{kpc}\right)^{2}$ & 39 \\
Cen X-4 & $(1.6 \pm 0.6)\left(d_{0} / 1.2 \mathrm{kpc}\right)^{2}$ & 4.6 \\
4U 1608-522 & $(30.0 \pm 15.2)\left(d_{0} / 3.6 \mathrm{kpc}\right)^{2}$ & 56 \\
4U 2129+47 & $\left(0.7_{-0.3}^{+1.5}\right)\left(d_{0} / 1.5 \mathrm{kpc}\right)^{2}(0.5-3.0 \mathrm{keV})$ & 1.6 \\
4U 2129+47 & $\left(5.6_{-3}^{+12}\right)\left(d_{0} / 6.0 \mathrm{kpc}\right)^{2}(0.5-3.0 \mathrm{keV})$ & 13 \\
\hline
\end{tabular}

${ }^{\text {a }}$ A range of $\left(N_{\mathrm{H}}, d_{0}\right)$ is assumed; See $\S \mathrm{A} 7$.

that the best-fit spectrum produced both observed spectra. Assuming that $r_{e}$ and $N_{\mathrm{H}}$ are the same between the two observations, the effective temperature would have to have dropped from $0.091 \pm 0.006 \mathrm{keV}(90 \%)$ to $0.081 \pm 0.002 \mathrm{keV}$ to explain the different spectra. The thermal timescale at the depth where the crust heating occurs is about a year (Brown et al. 1998); the decrease in effective temperature could be a signature of a cooling crust. Another possible explanation is that the $N_{\mathrm{H}}$ was lower during the 1992 observation than the 1994 observation; for example if $N_{\mathrm{H}, 22}=0.10$ during the HRI observation and $N_{\mathrm{H}, 22}=0.28$ during the 1994 observation, but with the same underlying $\mathrm{H}$ atmosphere spectrum, this would account for the observed difference in flux. In addition, if accretion is active during quiescence, the different X-ray spectra may reflect a decrease in the accretion rate by a factor $(0.081 / 0.0906)^{4}=0.64$, assuming a thermal spectrum.

With the quality of spectra we have here, we cannot definitely state the cause of the discrepancy between the 1992 and 1994 observations, but it is consistent with either a difference in the intervening $N_{\mathrm{H}}$, a difference in the effective temperature, time-variable accretion, or a combination of the three.

\section{REFERENCES}

Alcock, C. \& Illarionov, A. 1980, ApJ, 235, 534

Arnaud, K. A. 1996, in ASP Conf. Proc. 101, Astronomical Data Analysis Software and Systems V, ed. G. Jacoby \& J. Barnes (San Francisco: ASP), 17

Asai, K., Dotani, T., Hoshi, R., Tanaka, Y., Robinson, C. R., \& Terada, K. 1998, PASJ, 50, 611

Asai, K., Dotani, T., Kunieda, H., \& Kawai, N. 1996a, PASJ, 48, L27

Asai, K., Dotani, T., Mitsuda, K., Hoshi, R., Vaughan, B., Tanaka, Y., \& Inoue, H. 1996b, PASJ, 48, 257

Barret, D., McClintock, J. E., \& Grindlay, J. E. 1996, ApJ, 463, 963

Beekman, G., Shahbaz, T., Naylor, T., Charles, P. A., Wagner, R. M., \& Martini, P. 1997, MNRAS, 290, 303

Bildsten, L., \& Brown, E. F. 1997, ApJ, 477, 897

Bildsten, L., Salpeter, E. E., \& Wasserman, I. 1992, ApJ, 384, 143

Brown, E. F., Bildsten, L., \& Rutledge, R. E. 1998, ApJ, 504, L95

Callanan, P. J., Filippenko, A. V., \& Garcia, M. R. 1999, IAU Circ. 7086

Campana, S., Colpi, M., Mereghetti, S., Stella, L., \& Tavani, M. 1998a, Astron. Astrophys. Rev., 8, 279

Campana, S., Stella, L., Mereghetti, S., Colpi, M., Tavani, M., Ricci, D., Fiume, D. D., \& Belloni, T. 1998b, ApJ, 499, L65

Casares, J., Charles, P. A., \& Marsh, T. R. 1995, MNRAS, 277, L45

Charles, P., Hassall, B., Sahu, K., Broadhurst, T., Lawrence, A., Taylor, A., \& Hacking, P. 1989, IAU Circ. 4794

Chen, W., Shrader, C. R., \& Livio, M. 1997, ApJ, 491, 312

Chevalier, C., \& Ilovaisky, S. A. 1990, A\&A, 238, 163

Chevalier, C., Ilovaisky, S. A., Motch, C., Pakull, M., \& Mouchet, M. 1989, A\&A, 217, 108

Chitre, D. M., \& Hartle, J. B. 1976, ApJ, 207, 592

Cool, A. M., Grindlay, J. E., Cohn, H. N., Lugger, P. M., \& Slavin, S. D. 1995, ApJ, 439, 695

Corbet, R. H. D., Asai, K., Dotani, T., \& Nagase, F. 1994, ApJ, 436, L15

Cowley, A. P., \& Schmidtke, P. C. 1990, AJ, 99, 678

Dickey, J. M., \& Lockman, F. J. 1990, ARA\&A, 28, 215

Ebisawa, K., et al. 1994, PASJ, 46, 375

Esin, A. A., Narayan, R., Cui, W., Grove, J. E., \& Zhang, S.-N. 1998, ApJ, 505,854

Garcia, M., McClintock, J. E., Narayan, R., \& Callanan, P. J. 1997, in Proc. 13th North American Workshop on Cataclysmic Variables, ed. S. Howell, E. Kuulkers, \& C. Woodward (San Francisco: ASP), 506

Garcia, M. R., \& Callanan, P. J. 1999, AJ, 118, 1390

Gonzalez-Riestra, R., Cassatella, A., Wamsteker, W., Shrader, C., Shore, S., \& Lund, N. 1991, IAU Circ. 5174

Greiner, J., Hasinger, G., Molendi, S., \& Ebisawa, K. 1994, A\&A, 285, 509

Grindlay, J. E., Cool, A. M., Callanan, P. J., Bailyn, C. D., Cohn, H. N., \& Lugger, P. M. 1995, ApJ, 455, L47

Haensel, P., \& Zdunik, J. L. 1990, A\&A, 227, 431

Hertz, P., \& Grindlay, J. E. 1983, ApJ, 267, L83

Hjellming, R., \& Rupen, M. 1995, Nature, 375, 464

Horne, K., et al. 1996, IAU Circ. 6406

Huang, M., \& Wheeler, J. C. 1989, ApJ, 343, 229

Illarionov, A. F., \& Sunyaev, R. A. 1975, A\&A, 39, 185

King, A. R., Kolb, U., \& Burderi, L. 1996, ApJ, 464, L127
McClintock, J. E. 1998, in AIP Conf. Proc. 431, Accretion Processes in Astrophysical Systems: Some Like it Hot!, ed. S. S. Holt \& T. R. Kallman (New York: AIP), 290

McClintock, J. E., Horne, K., \& Remillard, R. A. 1995, ApJ, 442, 358

McClintock, J. E., \& Remillard, R. A. 1986, ApJ, 308, 110

Menou, K., Esin, A. A., Narayan, R., Garcia, M. R., Lasota, J. P., \& MCClintock, J. E. 1999, ApJ, 520, 276

Mineshige, S., \& Wheeler, J. C. 1989, ApJ, 343, 241

Narayan, R., Barret, D., \& McClintock, J. E. 1997a, ApJ, 482, 448

Narayan, R., Garcia, M. R., \& McClintock, J. E. 1997b, ApJ, 478, L79

Oke, J. B., \& Greenstein, J. L. 1977, ApJ, 211, 872

Orosz, J. A., \& Bailyn, C. D. 1997, ApJ, 477, 876

Orosz, J. A., Bailyn, C. D., McClintock, J. E., \& Remillard, R. A. 1996, ApJ, 468, 380

Pavlov, G. G., \& Shibanov, I. A. 1978, Soviet Astron., 22, 214

Penninx, W., Damen, E., Van Paradijs, J., Tan, J., \& Lewin, W. H. G. 1989, A\&A, 208, 146

Predehl, P., \& Schmitt, J. H. M. M. 1995, A\&A, 293, 889

Rajagopal, M., \& Romani, R. W. 1996, ApJ, 461, 327

Rhoades, C. E., \& Ruffini, R. 1974, Phys. Rev. Lett., 32, 324

Romani, R. W. 1987, ApJ, 313, 718

Rutledge, R. E., Bildsten, L., Brown, E. F., Pavlov, G. G., \& Zavlin, V. E. 1999, ApJ, 514, 945

Schild, R. E. 1977, AJ, 82, 337

Shahbaz, T., Naylor, T., \& Charles, P. A. 1997, MNRAS, 285, 607

Shrader, C. R., Wagner, R. M., \& Starrfield, S. 1992, IAU Circ. 5591

Stella, L., White, N. E., \& Rosner, R. 1986, ApJ, 308, 669

Tanaka, Y., \& Lewin, W. 1995, in X-Ray Binaries, Vol. 1, ed. W. Lewin, J. Van Paradijs, \& E. Van Den Heuvel (Cambridge: Cambridge Univ. Press), 126

Tanaka, Y., \& Shibazaki, N. 1996, ARA\&A, 34, 607

Thomas, B., Corbet, R., Smale, A. P., Asai, K., \& Dotani, T. 1997, ApJ, 480 , L21

Thorstensen, J., Charles, P., Bowyer, S., Briel, U. G., Doxsey, R. E., Griffiths, R. E., \& Schwartz, D. A. 1979, ApJ, 233, L57

Tsunemi, H., Kitamoto, S., Okamura, S., \& Roussel-Dupre, D. 1989, ApJ, 337, L81

Ueda, Y., Inoue, H., Tanaka, Y., Ebisawa, K., Nagase, F., Kotani, T., \& Gehrels, N. 1998, ApJ, 492, 782; erratum ApJ, 500, 1069

Van der Klis, M. 1995, in X-Ray Binaries, Vol. 1, ed. W. Lewin, J. Van Paradijs, \& E. Van Den Heuvel (Cambridge: Cambridge Univ. Press), 252

Van Paradijs, J. 1996, ApJ, 464, L139

Van Paradijs, J., Verbunt, F., Shafer, R. A., \& Arnaud, K. A. 1987, A\&A, 182,47

Verbunt, F., Belloni, T., Johnston, H. M., Van der Klis, M., \& Lewin, W. H. G. 1994, A\&A, 285, 903

Verbunt, F., Bunk, W., Hasinger, G., \& Johnston, H. M. 1995, A\&A, 300, 732

Verbunt, F., Elson, R., \& Van Paradijs, J. 1984, MNRAS, 210, 899

Wagner, R. M., Bertram, R., Starrfield, S. G., Howell, S. B., Kreidl, T. J., Bus, S. J., Cassatella, A., \& Fried, R. 1991, ApJ, 378, 293 
Wagner, R. M., Kreidl, T. J., Howell, S. B., \& Starrfield, S. G. 1992, ApJ, 401, L97

Wagner, R. M., Starrfield, S. G., Hjellming, R. M., Howell, S. B., \& Kreidl, T. J. 1994, ApJ, 429, L25

Wu, C.-C., Aalders, J. W. G., Van Duinen, R. J., Kester, D., \& Wesselius, P. R. 1976, A\&A, 50, 445
Zampieri, L., Turolla, R., Zane, S., \& Treves, A. 1995, ApJ, 439, 849 Zavlin, V. E., Pavlov, G. G., \& Shibanov, Y. A. 1996, A\&A, 315, 141 Zavlin, V. E., Pavlov, G. G., \& Truemper, J. 1998, A\&A, 331, 821 Zavlin, V. E., Trümper, J., \& Pavlov, G. G. 1999, ApJ, 525, 959

Zhang, S. N., Yu, W., \& Zhang, W. 1998a, ApJ, 494, L71 